\title{
Tyramine and Octopamine Independently Inhibit Serotonin- Stimulated Aversive Behaviors in Caenorhabditis elegans through Two Novel Amine Receptors
}

\author{
Rachel T. Wragg, Vera Hapiak, Sarah B. Miller, Gareth P. Harris, John Gray, Patricia R. Komuniecki, and \\ Richard W. Komuniecki \\ Department of Biological Sciences, University of Toledo, Toledo, Ohio 43606-3340
}

\begin{abstract}
Biogenic amines modulate key behaviors in both vertebrates and invertebrates. In Caenorhabditis elegans, tyramine (TA) and octopamine (OA) inhibit aversive responses to $100 \%$, but not dilute (30\%) octanol. TA and $0 \mathrm{~A}$ also abolish food- and serotonin-dependent increases in responses to dilute octanol in wild-type but not tyra-3(ok325) and f14d12.6( ok371) null animals, respectively, suggesting that TA and OA modulated responses to dilute octanol are mediated by separate, previously uncharacterized, G-protein-coupled receptors. TA and OA are high-affinity ligands for TYRA-3 and F14D12.6, respectively, based on their pharmacological characterization after heterologous expression. f14d12.6::8fp is expressed in the ASHs, the neurons responsible for sensitivity to dilute octanol, and the sra-6dependent expression of F14D12.6 in the ASHs is sufficient to rescue OA sensitivity in f14d12.6(ok371) null animals. In contrast, tyra-3::gfp appears not to be expressed in the ASHs, but instead in other neurons, including the dopaminergic CEP/ADEs. However, although dopamine (DA) also inhibits 5-HT-dependent responses to dilute octanol, TA still inhibits in dop-2; dop-1; dop-3 animals that do not respond to DA and cat-2(tm346) and Pdat-1::ICE animals that lack significant dopaminergic signaling, suggesting that DA is not an intermediate in TA inhibition. Finally, responses to TA and $0 \mathrm{~A}$ selectively desensitize after preexposure to the amines. Our data suggest that although tyraminergic and octopaminergic signaling yield identical phenotypes in these olfactory assays, they act independently through distinct receptors to modulate the ASH-mediated locomotory circuit and that $C$. elegans is a useful model to study the aminergic modulation of sensory-mediated locomotory behaviors.
\end{abstract}

Key words: olfaction; tyramine; adaptation; behavior; C. elegans; octopamine

\section{Introduction}

Classical biogenic amines, such as serotonin (5-HT) and dopamine (DA) play important roles in synaptic plasticity and modulate many key behaviors (Lucki, 1998; Berke and Hyman, 2000). Recently, trace amines, such as tyramine (TA), octopamine (OA), and $\beta$-phenylethylamine have also been implicated in a number of behavioral disorders, including depression, schizophrenia, and attention deficit hyperactivity disorder (ADHD). A family of trace amine-associated receptors (TAARs) has been identified; however, to date, the endogenous ligands for only a few of these receptors have been characterized (Borowsky et al., 2001). More importantly, the potential physiological roles of trace amines has been only cursorily described. In contrast to vertebrates, tyraminergic and octopaminergic signaling has been much better characterized in invertebrates. In nematodes, TA and OA gener-

Received Aug. 1, 2007; revised 0ct. 16, 2007; accepted 0ct. 16, 2007.

This work was supported by National Institutes of Health (NIH) Grant Al-145147 (R.W.K.) and funds from the Joan L. and Julius H. Jacobson Professorship. We thank Drs. Bruce Bamber and Rob Steven for critical discussion of this manuscript, Dr. Shohei Mitani at the National Bioresources Project, and the Caenorhabditis elegans Genetics Center, which is supported by NIH National Center for Research Resources for the strains used in these studies.

Correspondence should be addressed to Richard W. Komuniecki, Department of Biological Sciences, University of Toledo, 2801 West Bancroft Street, Toledo, OH 43606-3340. E-mail: rkomuni@uoft02.utoledo.edu.

D0l:10.1523/JNEUROSCI.3495-07.2007

Copyright $\odot 2007$ Society for Neuroscience 0270-6474/07/2713402-11\$15.00/0 ally oppose the action of 5-HT. For example, in Caenorhabditis elegans, exogenous TA or OA causes hyperactivity and suppresses 5-HT-stimulated pharyngeal pumping and egg laying (Horvitz et al., 1982; Ségalat et al., 1995; Niacaris and Avery, 2003; Rex et al., 2004; Alkema et al., 2005).

The present study was designed to characterize the amines, receptors, and neurons involved in the OA/TA modulation of a simple, sensory-mediated locomotory behavior, octanol avoidance, using the C. elegans model system. C. elegans is an excellent model to study the aminergic modulation of locomotory behavior. In addition to its simple nervous system and well characterized olfactory preferences, a variety of amine-dependent behaviors have been identified, which include locomotion, feeding, aversive learning, and olfaction, and null alleles are available for most of its predicted biogenic amine (BA) G-protein-coupled receptors (GPCRs). C. elegans can detect both volatile and watersoluble chemicals through 12 different sensory neurons, and bacteria or 5-HT can modulate sensitivity to a variety of different odorants (de Bono and Maricq, 2005). For example, the ASH sensory neuron is essential for aversive responses to dilute octanol, and this response is dramatically increased in the presence of bacteria or 5-HT (Chao et al., 2004).

In the present study, we have demonstrated that OA, TA, and DA each independently abolish 5-HT-dependent increases in 
sensitivity to dilute octanol. OA and TA inhibition is mediated by two novel C. elegans OA and TA receptors: F14D12.6 is essential for inhibition by OA, and TYRA-3 is essential for inhibition by TA. Importantly, f14d12.6::gfp is expressed in the ASHs, the sensory neurons responsible for sensitivity to dilute octanol and sra-6 promoter-driven F14D12.6 expression is sufficient to rescue OA sensitivity in $f 14 d 12.6(\mathrm{ok} 371)$ null animals. As predicted, TYRA-3 and F14D12.6 exhibit highest affinity for TA and OA, respectively, based on pharmacological studies with heterologously expressed receptors in mammalian cells. Finally, responses to TA and OA selectively desensitize after preexposure to the amines. Our data suggest that although tyraminergic and octopaminergic signaling often yield identical phenotypes, they can be mediated by separate receptors located in distinct neurons and highlight the utility of the C. elegans model system to study the complexity of aminergic modulation of aversive olfactory behaviors.

\section{Materials and Methods}

Materials. DMEM was purchased from Mediatech (Herndon, VA). Both fetal bovine serum (FBS) and bovine calf serum (BCS) were purchased from Hyclone (Logan, UT). All cell culture plasticware was purchased from Sarstedt (Newton, NC). All cell lines were originally ordered from the American Type Culture Collection (Rockville, MD). Restriction enzymes were purchased from New England Biolabs (Beverly, MA) and Promega (Madison WI). [ $\left.{ }^{3} \mathrm{H}\right] \mathrm{LSD}(75 \mathrm{Ci} / \mathrm{mmol})$ was purchased from PerkinElmer (Wellesley, MA). All other chemicals were purchased from Sigma-Aldrich (St Louis, MO), including penicillin/streptomycin, trypsin/EDTA TA, DA and OA hydrochloride, 5-HT creatine sulfate complex, and 1-octanol. A C. elegans cDNA library was a gift from Robert Barstead (Oklahoma Medical Research Foundation, Oklahoma City, $\mathrm{OK})$. Green fluorescent protein (GFP) reporter constructs were obtained from Andy Fire (Stanford University, Palo Alto, CA).

Strains and reagents. General techniques for the culture and handling of worms have been described previously (Brenner, 1974). The Bristol N2 strain was used as wild type. Strains obtained from the Caenorhabditis Genetics Center (University of Minnesota, Minneapolis, MN) include dop-1(vs100), dop-2(vs105), dop-3(vs106), dop-2(v105); dop-1(vs100); dop-3(vs106), dat-1(tm903), gpa-1(pk15), gpa-7pk61), gpa-11(pk349), gpa-12(pk322), gpa-13(pk1270), gpa-14(pk342), gpa-15(pk477), gpa16(pk481), ser-2 (pk1357), ser-3(ad1774), tdc-1(n3419), tbh-1(n3247), tyra-2(tm1846), tyra-3(ok325), and f14d12.6(ok371). All strains were out-crossed at least five times before use. The double and triple mutants ser-2(pk1357); tyra-2(tm1846), ser-3(ad1774); f14d12.6(ok371), and tyra3(ok325); tyra-2(tm1846); ser-2(pk1357) were constructed using standard genetic techniques. lin-15(n765ts); akEx387(Pdat-1::GFP; Pdat-1::ICE) was a kind gift from V. Maricq (The University of Utah, Salt Lake City, UT) and cat-2(tm346) from J. Duerr (Ohio University, Athens, $\mathrm{OH}$ ).

Behavioral assays. All experiments used age-matched, well fed young adults grown at $20^{\circ} \mathrm{C}$ on standard nematode growth medium (NGM), seeded with Escherichia coli strain OP50. Octanol avoidance was assayed as described previously (Chao et al., 2004). Briefly, fourth-stage larvae were picked $24 \mathrm{~h}$ before testing and grown at $20^{\circ} \mathrm{C}$. NGM plates were prepared the morning of the experiment by adding 5-HT, TA, OA, and/or DA (final concentration, $4 \mathrm{~mm}$ ) to liquid NGM before pouring. Bacteria plates were prepared $24 \mathrm{~h}$ before assay. Dilute (30\%) 1-octanol was prepared daily using $100 \%$ ethanol (v/v). For assay, animals were placed on a transfer plate for $10 \mathrm{~min}$ to minimize carry over and then transferred to fresh test plates and incubated for $10 \mathrm{~min}$ (NGM), $20 \mathrm{~min}$ (bacteria), or $30 \mathrm{~min}$ (5-HT, DA, OA, TA) before assay. Five animals were placed on each plate, and the blunt end of a hair (Loew-Cornell series 8 paintbrush) dipped in octanol was placed in front of a forwardmoving animal. The time taken to reverse during a $20 \mathrm{~s}$ exposure to octanol was recorded. Animals that failed to reverse in $20 \mathrm{~s}(<15 \%)$ were not included in the analysis, because wild-type C. elegans spontaneously reverse on average every $20 \mathrm{~s}$ (Chao et al., 2005).
Adaptation assays were based on those described by Bargmann et al. (1993). NGM plates were prepared as described above. Age-matched young adults were incubated on a transfer plate for $10 \mathrm{~min}$ before transfer to a $1.6 \mathrm{ml}$ microcentrifuge tube containing $\mathrm{S}$ basal $(1 \mathrm{ml})$ and washed three times. Then, $\mathrm{S}$ basal $(1 \mathrm{ml})$ containing either TA or OA $(4 \mathrm{~mm})$ was added, and the animals were incubated for up to $6 \mathrm{~h}$ at $20^{\circ} \mathrm{C}$. Control animals were incubated under the same conditions in the absence of ligand. After incubation, the animals were placed on NGM plates for 10 min and assayed as described above. Data are presented as mean \pm SEM $(n)$ and analyzed by two-tailed Student's $t$ test. $p$ values are indicated as follows: ${ }^{*} p<0.05 ;{ }^{* *} p<0.01 ;{ }^{* * *} p<0.001$.

Cloning of tyra- 3 and f14d12.6. To clone the tyra- 3 cDNA for expression, specific primers (TYRA-3F and TYRA-3R) were designed against the tyra-3 open reading frame (TYRA-3F: 5' -ATATAAGCTTATGGCGGCGCTTGGCGGC-3'; TYRA-3R: 5'-TCTAGATTAGAATATTGTCGATTGCTGATGAAGTTTCTTG-3'). Restriction sites for additional subcloning are italicized, and the start and stop codons are underlined. Using a C. elegans cDNA library prepared from mixed stage animals as a template, an $\sim 1.7 \mathrm{~kb}$ band was obtained, isolated, and subcloned into the PCR 2.1 vector (Invitrogen, Carlsbad, CA). The $\sim 1.7 \mathrm{~kb}$ cDNA was then subcloned into pFLAG-CMV2 (Sigma-Aldrich).

To clone the f14d12.6 cDNA, specific primers were designed against the predicted f14d12.6 open reading frame (F14F: $5^{\prime}-G A A G A T$ CTATGTGGAACCTTAACTGC-3' and F14R: 5'-TCCCCGCGGTCATTTGTAGAACTCC- $3^{\prime}$ ). Italicized nucleotides represent the restriction sites BglII and SacII, respectively, and underlined nucleotides represent start and stop codons. Amplified PCR products were run on 1\% agarose gels. Bands of correct size were purified and cloned into pGEM vector (Promega). Finally, the $\sim 1.3 \mathrm{~kb}$ f14d12.6 cDNA was subcloned into pDisplay (Invitrogen) using the 5' BglII and 3' SacII restriction sites. Insertion of $f 14 d 12.6 \mathrm{cDNA}$ into the MCS of the vector allowed for the production of an N-terminal hemagglutinin-tagged receptor.

Expression of TYRA-3 and F14D12.6 in mammalian cell lines. To express TYRA-3, a pFLAG-tyra-3 construct was transiently transfected into COS-7 cells using Lipofectamine 2000 (Invitrogen) (at a DNA:Lipofectamine ratio of $1 \mu \mathrm{g}: 3 \mu \mathrm{l}$ ). Cells were harvested $48 \mathrm{~h}$ after transfection, and crude membrane preparations were prepared as described previously (Rex and Komuniecki, 2002). Briefly, COS-7 cells were washed with ice-cold PBS and incubated in $15 \mathrm{~mm}$ Tris- $\mathrm{HCl}, \mathrm{pH} 7.4,1 \mathrm{~mm}$ EDTA, $1 \mathrm{~mm}$ phenylmethylsulfonyl fluoride (PMSF) at $4^{\circ} \mathrm{C}$ for $10 \mathrm{~min}$. Cells were scraped from the plates and centrifuged at $4000 \times g$ for $15 \mathrm{~min}$, and the pellet was resuspended in ice-cold TEM (Tris- $\mathrm{HCl}, \mathrm{pH} 7.4,10$ mм $\mathrm{MgCl}_{2}, 0.5$ mм EDTA) containing $1 \mathrm{~mm}$ PMSF. Cells were lysed on ice by sonication and centrifuged at $20,000 \times g$ for $1 \mathrm{~h}$ at $4^{\circ} \mathrm{C}$. The pellet was homogenized in TEM buffer.

To express F14D12.6, the pDisplay-f14d12.6 constructs were transiently transfected into COS-7, NIH-3T3, CHO, and HEK293t cells $\left(\sim 5 \times 10^{5}\right.$ cells per $10 \mathrm{~cm}$ dish) using Lipofectamine 2000 (Invitrogen), as described above. The transfected cells were incubated at $37^{\circ} \mathrm{C}$ for $24 \mathrm{~h}$ after transfection and then moved to a humidified $28^{\circ} \mathrm{C}, 3 \% \mathrm{CO}_{2}$ incubator for an additional $24 \mathrm{~h}$ before harvesting for membrane preparation. This temperature shock was essential for expression. To change glutamine to a leucine in the F16D12.6 sequence to conform with a consensus endoplasmic reticulum exit motif (F(X6)I/L I/L), the GeneTailor site-directed mutagenesis system was used per the manufacturer's instructions (Invitrogen). The following primers were used for mutagenesis: F14 Mut-5' (TTCAAACGGGGTCTTTGCAAACtGCTGCACAAAT) and F14 Mut-3' (TTTGCAAAGACCCCGTTTGAATTCACGATTGAA). The mutagenized nucleotide that changes the codon from a glutamine to a leucine is indicated in lower case. Protein concentration was determined by Bradford assay (Bio-Rad Laboratories, Hercules, CA).

Membrane preparation and radioligand binding assays. Radioligand binding assays were performed as previously described (Huang et al., 2002; Hobson et al., 2003). Briefly, saturation binding was determined using crude membranes (15 $\mu \mathrm{g}$ of protein) incubated in various concentrations of $\left[{ }^{3} \mathrm{H}\right] \mathrm{LSD}(1-50 \mathrm{~nm})$, to obtain $B_{\max }$ and $K_{\mathrm{d}}$ values. For inhibition assays, membranes (10 $\mu$ g of protein) were incubated with various concentrations of ligand and $20 \mathrm{~nm}\left[{ }^{3} \mathrm{H}\right] \mathrm{LSD}$. Total and nonspecific binding was determined in the absence and presence of 1000-fold excess 
unlabeled LSD, respectively. All saturation and inhibition binding assays were performed at room temperature after $1 \mathrm{~h}$ in the dark and terminated by filtration onto 96-well microplates with GF/B filters (PerkinElmer) previously soaked with $0.3 \%$ polyethyleneimine. Filters were washed (three times) with ice-cold TEM and dried overnight, and radioactivity was determined by liquid scintillation counting. All radioligand binding data were analyzed by nonlinear regression analysis using Deltagraph (Deltagraph, version 4.05e; Deltapoint, Chicago, IL) and derived from at least three separate experiments performed in triplicate. An $F$ test was performed on the inhibition binding data to determine best fit for onesite or two-site binding models.

Localization of f14d12.6::gfp and rescue of f14d12.6(ok371) and tyra3(ok325) null animals. All rescue constructs were created by overlap fusion PCR (Hobert, 2002). To create the full-length f14d12.6::gfp translational fusion with GFP sequence inserted into the predicted third intracellular loop of the receptor, three different PCR fragments were fused by two rounds of PCR. Primers were used to amplify a $3987 \mathrm{bp}$ fragment upstream from the start ATG and the $5^{\prime}$ end of the f14d12.6 genomic sequence including the first $53 \mathrm{nt}$ of exon $\mathrm{V}$ [promoter (prom) forward (F), AGTTTTTTCCACCAATATTTTCCGCTT; F14 prom reverse (R) + GFP, AGTGAAAAGTTCTTCTCCTTTACTCATTCGAAGCTGCGAACATACTTTGTTAAT]. A second primer set was used to amplify the $3^{\prime}$ end of the gene including the remaining nucleotides of exon $\mathrm{V}$ and $\sim 360$ nt downstream of the $3^{\prime}$ untranslated region (UTR) (5'NheI, GCTAGCAAAATATCCGAAGAATTGCCAAGACAA; $3^{\prime}$ UTR BspEI, TCCGGAGCCTTCCCTATAAAAGCCTTTTTA). The italicized nucleotides indicate NheI and BspEI restriction sites, respectively, that were used to subclone the $3^{\prime}$ end of f14d12.6 into the GFP expression vector pPD117.01. The sequence for GFP, inserted into exon V, and the $3^{\prime}$ end of the gene were amplified using the following primers: GFP F, ATGAGTAAAGGAGAAGAACTTTTCACTGGAG; F14 3'R, GCCTTCCCTATAAAAGCCTTTTTA. The $5^{\prime}$ fragment of the f14d12.6 promoter contained a 54 nt overlap with GFP, whereas the $3^{\prime}$ fragment was amplified from the pPD117.01 vector containing GFP and the remainder of the f14d12.6 sequence. Last, these two products were combined and amplified using the primers F14 prom F and F14 $3^{\prime} \mathrm{R}$ to create a full-length f14d12.6 with the $3^{\prime}$ UTR, and GFP in the predicted third intracellular loop of the receptor. Using this construct, the FY745 f14d12.6(ok371) grEx157[Pf14d12.6::f14d12.6::gfp] transgenic line was created, as described below.

To express F14D12.6 in the ASH sensory neurons, the sra- 6 promoter and the f14d12.6 cDNA with sequence coding for GFP inserted into the predicted third intracellular loop were created by overlap fusion PCR. The sra-6 promoter is expressed in the ASH, as well as the ASI and PVQ neurons (Troemel et al., 1995). The sra-6 promoter was amplified using (prom F, CAGACGGTGGTATGCAACCAATG; prom F14 R, CACTGCAGTTAAGGTTCCACATACAACTTA) as described above. Using this construct, the FY746 f14d12.6(ok371); grEx158[Psra-6::f14d12.6] transgenic line was created, as described below.

To create a full-length tyra- 3 transgene that included the full-length tyra-3 gene and $3^{\prime}$ UTR, primers were designed to amplify $\sim 10 \mathrm{~kb}$ upstream of the predicted TYRA-3 ATG (TYRA-3 F, CGCTTACTGGTTTTATTCGCTGAC; TYRA-3 R, CCCGGTTGTTTGAAAAAAGTTTCAC). Using this construct, the FY747 tyra-3(ok325) grEx159[Ptyra-3::tyra-3] transgenic line was created, as described below.

PCR products were pooled from at least three separate PCRs (1 ng total) and were coinjected with either myo-3::gfp or rol-6 plasmid (pRF4) and carrier DNA into gonads of $C$. elegans wild-type or null mutant animals by standard techniques (Kramer et al., 1990; Mello and Fire, 1995). At least five transformed lines were analyzed.

Derivation of phylogenetic trees. BA receptor protein sequences were downloaded from NCBI and abridged to the operational taxonomic unit comprising the seven transmembrane regions; specifically, the $\mathrm{N}$ termini were deleted to include 18 a before the first conserved amino acids in transmembrane domain I (TM I; N1.50), the third intracellular loop was deleted 18 aa after P5.50 (TM V) and 17 aa before F6.50 (TM VI), and the $\mathrm{C}$ termini were truncated to 20 aa after P7.50 (TM VII). Annotated sequences were initially aligned using the ClustalW algorithm within MegAlign (DNAStar, Madison, WI) using default parameters (pairwise
A

B
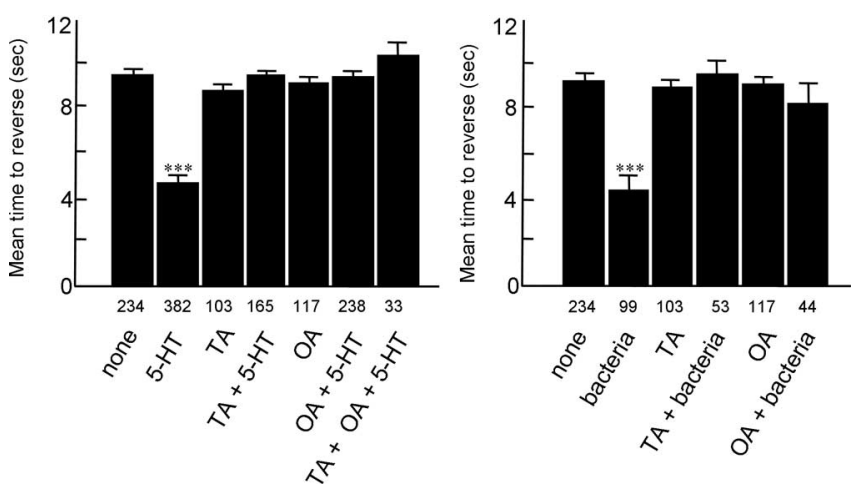

Figure 1. Both tyramine and octopamine abolish both food-and 5-HT-dependent responses to dilute octanol. $\boldsymbol{A}$, Wild-type animals off food were examined for their ability to respond to dilute octanol (30\%) in the presence or absence of exogenous $5-\mathrm{HT}, \mathrm{TA}$, or OA (4 mM), as described in Materials and Methods. B, Wild-type animals on food (E. coliOP50) were examined for their ability to respond to dilute octanol (30\%) in the presence or absence of exogenous TA or $0 \mathrm{~A}(4 \mathrm{~mm})$. Data are presented as mean \pm SE and analyzed by a two-tailed Student's $t$ test. ${ }^{* * *} p<0.001$, significantly different from wild-type animals under identical incubation conditions. The number of trials is indicated under each bar.

and multiple gap opening penalty of 10 , and gap extension penalties of 0.1 and 0.2 , respectively) and finalized using manual adjustment (all alignments available upon request). The statistical reliability of tree branching was assessed using bootstrap analysis (1000 replicates with random seed), and trees were compiled using the Phylogenetic Alignment Utility Program (PAUP, version 10.3). Trees were obtained using an optimality criterion of maximal parsimony and a neighbor-joining search algorithm. Accession numbers of GPCRs used in the alignment are listed in the legend to Figure 3.

\section{Results}

\section{Both TA and OA abolish 5-HT-dependent increases in} responsiveness to dilute octanol

The role of feeding status and 5-HT in the modulation of octanol avoidance has been demonstrated previously (Chao et al., 2004). For example, when animals are on food, the ASH sensory neurons are essential for responses to dilute (30\%) octanol. More importantly, both 5-HT and the presence of bacteria dramatically increase responsiveness (i.e., decrease time taken to reverse) to dilute octanol through a pathway requiring GPA-11, a G-protein expressed exclusively in the ASH and ADL sensory neurons, suggesting that 5-HT directly alters ASH sensitivity. To more fully characterize the amines and receptors involved in the modulation of ASH-mediated locomotory behaviors, we examined the effects of TA and OA on the 5-HT-dependent increase in responsiveness to dilute octanol (Fig. 1). As observed for other 5-HT-dependent phenotypes, incubation of wild-type animals in either exogenous TA or OA (4 mM) completely abolished food- or 5-HT (4 mM)dependent increases in responsiveness to dilute octanol. In addition, $t d c-1(n 3419)$ animals that contain no TA or OA or $t b h-$ 1(n3247) null animals that contain no OA both exhibited more rapid responses to dilute octanol than wild-type animals, suggesting that endogenous OA may normally modulate octanol responsiveness (Fig. 2) (Alkema et al., 2005). However, in the presence of exogenous TA or OA, $t d c-1(n 3419), t b h-1(n 3247)$ and wildtype animals responded identically to dilute octanol ( $\sim 10 \mathrm{~s}$ ) (Fig. 2 ), suggesting that both TA and OA were involved. Interestingly, tbh-1(n3247) animals appear to contain significantly higher TA levels than wild-type animals, and it is unclear why exogenous TA inhibits 5-HT-dependent increases in octanol sensitivity in these 


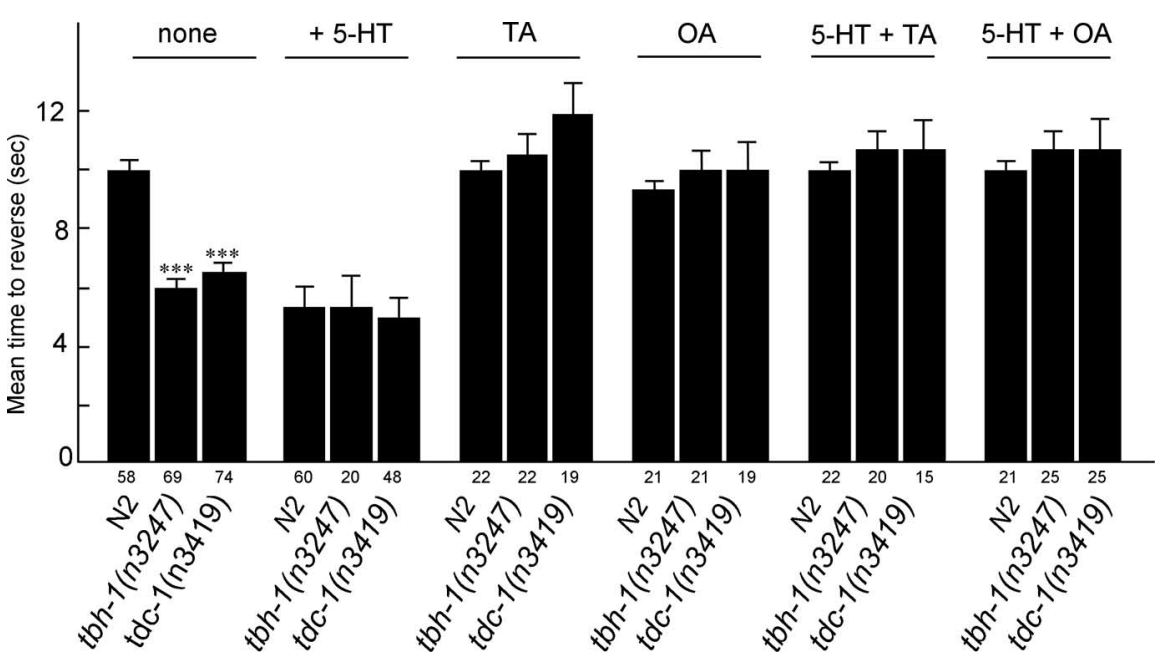

Figure 2. $\quad t d c-1$ (n3419) and $t b h-1$ (n3247) animals exhibit elevated basal responses to dilute octanol. The responses of wildtype and TA/OA mutant animals to dilute (30\%) octanol were examined in the absence of food and/or in the presence of either exogenous TA or OA ( $4 \mathrm{~mm}$ ), as described in Materials and Methods. Data are presented as mean $\pm S E$ and analyzed by a two-tailed Student's $t$ test. ${ }^{* * *} p<0.001$, significantly different from wild-type animals under identical incubation conditions. The number of trials is indicated under each bar.

tbh-1(n3247) animals but the elevated endogenous TA levels do not (Alkema et al., 2005). Presumably, the additional TA in the tbh-1 animals is present in octopaminergic, not tyraminergic, neurons and may not be released physiologically. Alternatively, Chao et al. (2004) have demonstrated that precise 5-HT levels are required for the modulation of octanol sensitivity (either too much or too little 5-HT yields an aberrant response), and precise TA concentrations or localization also may be necessary for effective tyraminergic signaling.

TA and OA act through separate receptors to abolish 5-HTdependent increases in responsiveness to dilute octanol

Two C. elegans $\mathrm{G} \alpha_{\mathrm{o}}$-coupled TA receptors (SER-2, TYRA-2) and a predicted $\mathrm{G} \alpha_{\mathrm{q}}$-coupled OA receptor (SER-3) have been described previously (Rex and Komuniecki, 2002; Rex et al., 2005; Suo et al., 2006). To determine whether these TA/OA receptors were involved in the TA/OA effects on octanol sensitivity described above, ser-2(pk1357), tyra-2(tm1846), and ser-3(ad1774) null animals were examined for the aminergic sensitivity of their responses to dilute octanol. Surprisingly, TA and OA still abolished 5-HT-dependent increases in octanol sensitivity in each of these null mutants, although at least one of these receptors, TYRA-2, is expressed directly in the ASH sensory neuron (Rex et al., 2005). In addition, TA still significantly inhibited 5-HTdependent increases in octanol sensitivity in tyra-2; ser-2 double mutants, although the level of inhibition was significantly less than that observed in wild-type animals (see Fig. 4). The role of these two receptors in the partial inhibition is unclear and, as with the analyses of all null mutants, could be direct (i.e., modify signaling from the octanol sensing neurons) or indirect (i.e., proper neuronal developmental or axonal migration). Most importantly, these data suggested that additional, as yet uncharacterized, TA and OA receptors were involved in mediating the effects of these amines on olfaction.

Therefore, we used a bioinformatics approach to identify additional genes encoding previously uncharacterized TA/OAspecific GPCR BA receptors in the C. elegans genome (Fig. 3). Interestingly, all of the receptors identified using this approach clustered based on their documented G-protein coupling and to a lesser extent, ligand binding (Fig. 3). The ligand binding and potential coupling of many of these receptors have been characterized previously by analyses of null mutants and/or after heterologous expression in mammalian cells. They include one OA, two TA/OA, three 5-HT, and four DA receptors (Olde and McCombie, 1997; Hamdan et al., 1999; Rex and Komuniecki, 2002; Suo et al., 2002, 2003; Hobson et al., 2003; Tsalik et al., 2003; Chase et al., 2004; Sanyal et al., 2004; Rex et al., 2005). Most importantly, two of these putative BA receptor genes appeared to encode previously uncharacterized TA/OA receptors (tyra-3 and f14d12.6) based on extensive sequence comparisons (K. A. Smith and R. W. Komuniecki, unpublished results). TYRA-3 clustered most closely with a recently identified TA-specific insect GPCR that activates $\mathrm{Ca}^{2+}$-dependent chloride channels after expression in Xenopus oocytes (Fig. 3) (Cazzamali et al., 2005).

To determine whether these two putative TA/OA receptors were involved in the TA/OA inhibition of 5-HT-mediated increases in octanol sensitivity, we examined tyra-3(ok325) and f14d12.6(ok371) putative null mutant animals. f14d12.6(ok371) has a $1072 \mathrm{bp}$ deletion that removes a portion of exons I-VI and predicted TMs I-V, whereas tyra-3(ok325) has a 781 bp deletion that removes portions of exons IV-V and TMs III-V. Therefore, both f14d12.6(ok371) and tyra-3(ok325) are predicted null alleles, because these TMs are essential for ligand binding (Strader et al., 1989; Choudhary et al., 1993; Almaula et al., 1996; Roth et al., 1997). Under standard conditions, tyra$3(\mathrm{k} 325)$ and $f 14 d 12.6$ (ok371) animals exhibited no gross behavioral defects with respect to locomotion, feeding, or egg retention. In contrast, tyra-3(ok325) animals failed to respond to TA in assays of 5-HT-mediated increases in sensitivity to dilute octanol but exhibited a wild-type response to OA, whereas f14d12.6(ok371) null mutants failed to respond to OA but exhibited a wild-type response to TA (Fig. 4). As predicted, the lack of response to TA and OA in tyra-3(ok325) and f14d12.6(ok371) animals, respectively, could be rescued by the expression of either a full-length tyra-3 genomic construct that included $10 \mathrm{~kb}$ upstream of the predicted ATG or a full-length $f 14 d 12.6:: g f p$ translation fusion that included $4 \mathrm{~kb}$ upstream of the ATG and sequence for GFP inserted into the predicted third intracellular loop of the receptor (Fig. 5). These data strongly suggest that although both TA and OA oppose the action of 5-HT, their effects are mediated by distinct receptors and signaling pathways.

Aminergic signaling in sensory neurons is often mediated by specific heterotrimeric G-proteins. For example, 5-HTdependent increases in sensitivity to dilute octanol require a novel G $\alpha$ subunit, GPA-11, expressed exclusively in the ASH and ADL sensory neurons (Chao et al., 2004). Therefore, we examined mutant strains lacking each of the nonclassical $\mathrm{G} \alpha$ subunits previously demonstrated to be expressed in sensory neurons for TA and OA inhibition of 5-HT-dependent increases in sensitivity to dilute octanol [gpa-1(pk15); gpa-7(pk610); gpa-11(pk349); gpa-12(pk322); gpa-13(pk1270); gpa-14(pk342); gpa-15(pk477); $g p a-16(p k 481)]$. This analysis confirmed the importance of gpa-11 in 5-HT sensitization but failed to identify any additional 
nonclassical $\mathrm{G} \alpha$ subunits that might be involved in the TA/OA modulation of octanol sensitivity (data not shown).

TYRA-3 and F14D12.6 bind TA and OA, respectively, with high affinity

The results described above suggest that TYRA-3 and F14D12.6 bind TA and OA, respectively, with high affinity. To confirm these observations, both receptors were transiently expressed in mammalian COS-7 cells, and isolated membranes were examined for $\left[{ }^{3} \mathrm{H}\right] \mathrm{LSD}$ binding, as we have described for the pharmacological characterization of two other C. elegans TA receptors, SER-2 and TYRA-2 (Fig. 6) (Rex and Komuniecki, 2002; Rex et al., 2005). Culture systems for nematode cells are not available. However, several nematode GPCRs have been successfully expressed in mammalian cells, and, more importantly, their ligand-dependent coupling to particular second messenger pathways agrees with predictions from multiple sequence alignments and in vivo data, suggesting that G-proteins are highly conserved and that the characterization of nematode receptors in this heterologous system is physiologically relevant. Membranes from COS-7 cells transiently expressing TYRA-3 exhibited saturable [ $\left.{ }^{3} \mathrm{H}\right]$ LSD binding with a $K_{\mathrm{d}}$ and $B_{\max }$ of $31.1 \pm 4.7 \mathrm{~nm}$ and $5.4 \pm 1.3 \mathrm{pmol} / \mathrm{mg}$, respectively. As predicted, membranes from untransfected COS-7 cells did not bind LSD with high affinity (data not shown). More importantly, $\left[{ }^{3} \mathrm{H}\right] \mathrm{LSD}$ binding was inhibited with higher affinity by TA than $\mathrm{OA}$ with apparent $\mathrm{IC}_{50}$ values of $0.7 \pm 0.01$ and $65 \pm 5 \mu \mathrm{M}$, respectively (Fig. 6). DA and 5-HT had little effect on $\left[{ }^{3} \mathrm{H}\right]$ LSD binding $\left(\mathrm{IC}_{50}\right.$ values $>100 \mu \mathrm{M}$; data not shown). In contrast, membranes from COS-7, HEK293, and NIH3T3 cells transiently expressing F14D12.6 did not bind $\left[{ }^{3} \mathrm{H}\right] \mathrm{LSD}$ with high affinity, and antiFLAG immunostaining of FLAG-tagged F14D12.6 indicated that the receptor was not highly expressed in those cell lines under these expression conditions (data not shown). Therefore, we tried a variety of additional approaches to achieve functional F14D12.6 expression. Unfortunately, the more phylogenetically related insect Sf9 cells could not be used for these studies, because they express an endogenous OA receptor (Näsman et al., 2002). F14D12.6 lacks an FX6L/IL/I motif in its C terminus that is conserved in many mammalian GPCRs and appears to be essential for efficient localization to the plasma membrane (Duvernay et al., 2004). Therefore, we mutated the F(X6)QL sequence in F14D12.6 to F(X6)LL. However, this mutagenesis had no apparent effect on receptor expression (data not shown). Similarly, the inclusion of the Ig $\kappa$-chain leader sequence from the pDisplay vector, which was essential for the optimal surface expression of the C. elegans SER-7, had no effect on the expression of F14D12.6 (Hobson et al., 2003) (data not shown). Interestingly, the surface expression of a nematode neuropeptide-specific GPCR relied on a temperature shift from 37 to $28^{\circ} \mathrm{C}$ after transfection (Kubiak et al., 2003). Indeed, after an identical temperature protocol, low levels of saturable $\left[{ }^{3} \mathrm{H}\right] \mathrm{LSD}$ binding to membranes from HEK293T cells transiently expressing F14D12.6 could be demonstrated, with an apparent $K_{\mathrm{d}}$ and $B_{\max }$ of $42 \mathrm{~nm}$ and 1.9 $\mathrm{pmol} / \mathrm{mg}$, respectively (Fig. 6). More importantly, $\left[{ }^{3} \mathrm{H}\right] \mathrm{LSD}$ binding was inhibited with higher affinity by $\mathrm{OA}$ than $\mathrm{TA}$, with apparent $\mathrm{IC}_{50}$ values of $46 \pm 6$ and $317 \pm 36 \mathrm{nM}$, respectively (Fig. 6). As predicted, no specific $\left[{ }^{3} \mathrm{H}\right] \mathrm{LSD}$ binding was observed in heat-shocked untransfected cells (data not shown). These results confirm that TYRA-3 and F14D12.6 are capable of high-affinity TA and OA binding, respectively. 


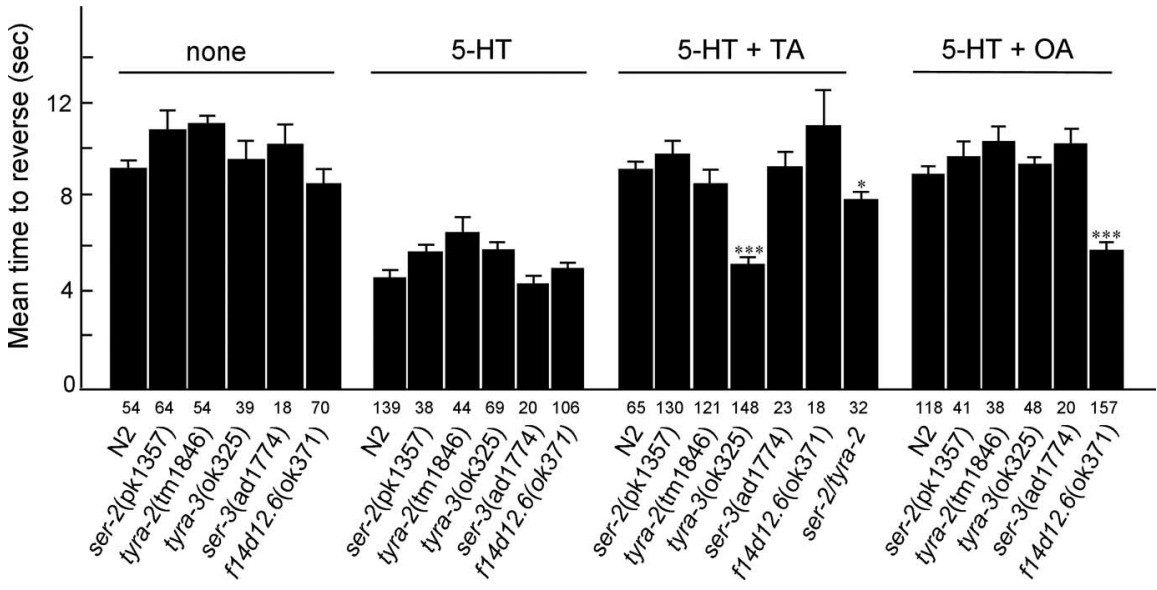

Figure 4. Tyramine and octopamine act through different receptors to abolish 5-HT-dependent responses to dilute octanol. C. elegans TA and $\mathrm{OA}$ receptor null mutant animals were examined for their ability to respond to dilute octanol in the presence or absence of 5-HT, TA, and/or OA (4 mm). Data are presented as mean \pm SE and analyzed by a two-tailed Student's $t$ test. ${ }^{*} p<0.05$ ${ }^{* * *} p<0.001$, significantly different from wild-type animals under identical incubation conditions. The number of trials is indicated under each bar.

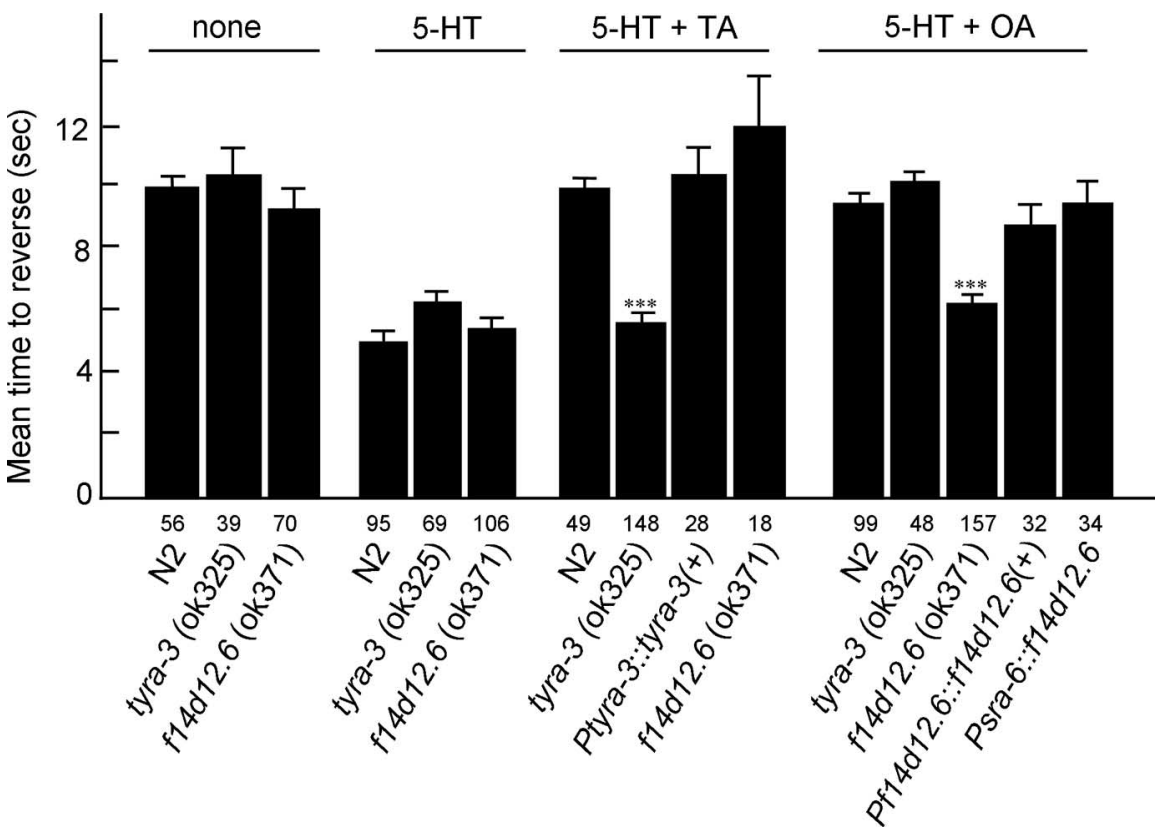

Figure 5. Rescue of tyramine and octopamine inhibition of 5-HT-dependent responses to dilute octanol in tyra-3(ok325) and f14d12.6 (ok371) null mutants. f14d12.6(ok371) or tyra-3(0k325) null mutants were rescued with the transgenes indicated below and assayed for responses to dilute octanol, as described in Materials and Methods. Either full-length f14d12.6::gfp $(+)$ (FY745f14 d12.6(ok371) grEx157[Pf14d12.6::f14d12.6::gfp]) or Psra-6::f14d12.6 (FY746 f14 d12.6(ok371); grEx158[Psra-6::f14d12.6]) transgenes rescued the $\mathrm{OA}$ inhibition of 5 -HT-dependent responses to dilute octanol in f14d12.7(ok371) null animals. Similarly, a full-length tyra-3 transgene, tyra-3(+) (FY747 tyra-3(ok325) grEx159[Ptyra-3::tyra-3]) rescued the TA inhibition of 5-HTdependent responses to dilute octanol. Data are presented as mean \pm SE and analyzed by a two-tailed Student's $t$ test. ${ }^{* * *} p<$ 0.001 , significantly different from wild-type animals under identical incubation conditions. The number of trials is indicated under each bar.

Localization of $f 14 d 12.6:: g f p$ and tyra-3::gfp expression and rescue of $f 14 d 12.6(\mathrm{ok} 371)$ null animals by F14D12.6 expression in the ASH sensory neurons

To examine $f 14 d 12.6$ expression, we created a full-length $f 14 d 12.6$ translational fusion with sequence encoding GFP inserted into the predicted third intracellular loop of the receptor. This approach was previously successful in creating a ser-7::gfp translational fusion that rescued ser-7(tm1325) null animals (Hobson et al., 2003). f14d12.6::gfp fluorescence was observed in a number of sensory neurons, including the ASHs, as well as a subset of head and tail neurons, the spermatheca and uterine toroid cells (Fig. 7). As described above, this f14d12.6::gfp transgene rescued the OA inhibition of 5-HT-stimulated increases in octanol sensitivity in $f 14 d 12.6$ (ok371) null mutants, strongly suggesting that this construct was functionally expressed. Indeed, OA sensitivity in f14d12.6(ok371) null animals could be fully rescued by the expression of a Psra-6::f14d12.6 transgene that restricts expression to the ASH and a small number of additional neurons, suggesting that F14D12.6 functions directly in the ASH to decrease sensitivity to octanol (Fig. 5). In contrast, tyra-3::gfp does not appear to be expressed in the ASHs or other sensory neurons, but instead in a subset of head and tail neurons, most robustly in the dopaminergic ADE/CEP neurons, as well as vulval muscle (Carre-Pierrat et al., 2006) (V. Hapiak, unpublished results).

DA is not involved in the TA inhibition of 5-HT-dependent responses to dilute octanol

As noted above, tyra-3::gfp is robustly expressed in the dopaminergic neurons and TYRA-3 clusters most closely with a TAspecific receptor that activates $\mathrm{Ca}^{2+}$ dependent chloride channels after expression in Xenopus oocytes, presumably through $\mathrm{G} \alpha_{\mathrm{q}}$ (Fig. 3) (Cazzamali et al., 2005) (V. Hapiak and R. W. Komuniecki, unpublished results). Therefore, we hypothesized that TA and TYRA-3 might stimulate the release of DA from the ADE/ CEPs, through the well described $\mathrm{G} \alpha_{\mathrm{q}}$ dependent pathways involved in the stimulation of neurotransmitter releases at the C. elegans neuromuscular junction (Mendel et al., 1995; Lackner et al., 1999; Miller et al., 1999; Nurrish et al., 1999). Indeed, DA also dramatically decreased 5-HTdependent increases in octanol sensitivity (Fig. 8). To determine whether the effects of TA might be mediated by increased DA release from the dopaminergic neurons, we examined the aminergic sensitivity of responses to dilute octanol in (1) cat2(tm346) null animals that lack tyrosine hydroxylase and exhibit dramatically reduced DA levels; (2) Pdat-1::ICE animals that lack dopaminergic neurons; and (3) dop-2; dop-1; dop-3 triple mutants that lack three key DA receptors (Chase et al., 2004; Sanyal et al., 2004; Nass et al., 2005). All three of these mutant strains exhibited more rapid reversals in response to dilute octanol than wild-type animals, suggesting that basal levels of DA may inhibit responses to dilute octanol (Fig. 8). In fact, these strains responded in $\sim 5 \mathrm{~s}$, identical to the responses of wild-type animals on food or in the presence of 5-HT (Fig. 8). In addition, DA had no effect on 5-HT-dependent increases in responses to 

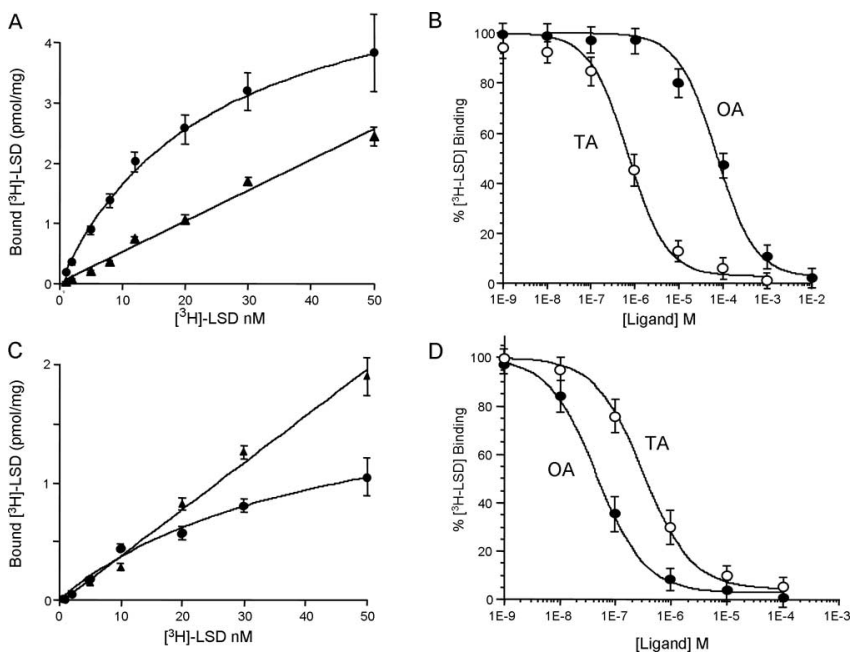

Figure 6. TYRA-3 and F14D12.6 bind tyramine and octopamine, respectively, with high affinity. Receptor expression and saturation and inhibition binding were conducted as described in Materials and Methods. $A, C,\left[{ }^{3} \mathrm{H}\right] \mathrm{LSD}$ binding to membranes isolated from $C O S-7$ cells expressing TYRA-3 $(\boldsymbol{A})$ and HEK293T expressing F14D12.6. Membranes prepared from COS-7 or HEK293T cells transiently transfected with TYRA-3 in pFLAG or F14D12.6 in pDisplay, respectively, were incubated with [ $\left.{ }^{3} \mathrm{H}\right] \mathrm{LSD}$ at concentrations ranging from 1 to $50 \mathrm{~nm}$ in the presence or absence of 1000-fold unlabeled LSD. Cells expressing F14D12.6 were temperature shocked at $28^{\circ} \mathrm{C}$ before membrane preparation, as described in Materials and Methods. Specific and nonspecific binding are represented by circles and triangles, respectively. $\boldsymbol{B}, \boldsymbol{D}$, Inhibition of $\left[{ }^{3} \mathrm{H}\right] \mathrm{LSD}$ binding to membranes from COS-7 and HEK293T cells expressing TYRA-3 (B) and F14D12.6 (D), respectively, prepared as described above. Data are representative of three independent experiments performed in triplicate.
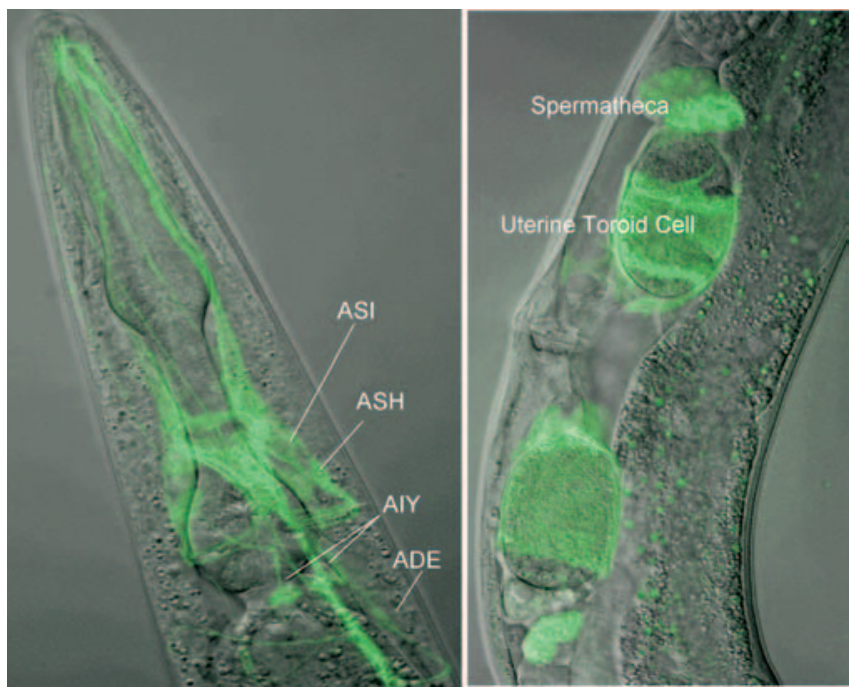

Figure 7. $\quad f 14 d 12.6:: g f p$ expression. A full-length $f 14 d 12.6:: g f p$ translational fusion comprising the entire $f 14 d 12.6$ gene with an additional $\sim 4 \mathrm{~kb}$ upstream from the predicted start codon and with sequence encoding GFP inserted into the predicted third intracellular loop was injected into f14d12.6(ok371) animals, and the transgenic animals (FY745 f14 d12.6(ok371) grEx157[Pf14d12.6::f14d12.6::gfp]) were examined for fluorescence by confocal microscopy. Left, GFP fluorescence in a number of amphidial neurons, including the ASI, ASH, AIY, and dopaminergic ADE/CEP neurons. Right, GFP fluorescence in the midbody region, including the spermatheca and uterine toroid cells.

dilute octanol in the $d o p-2 ; d o p-1 ; d o p-3$ animals, suggesting that the effects of DA were mediated by at least one of these three DA receptors. As predicted, a wild-type response to dilute octanol could be restored by preincubation of the cat-2(tm346) animals in DA. In contrast, TA still decreased responses to dilute octanol in cat-2(tm346), dop-2; dop-1; dop-3, and Pdat-1::ICE animals, strongly suggesting that responses to TA did not involve the modulation of DA release (Fig. 8).

\section{TA and OA also inhibit responses to $100 \%$ octanol}

In contrast to responses to dilute octanol, responses to $100 \%$ octanol are not sensitive to 5-HT or food, unless the ASH sensory neuron is ablated and octanol is sensed by additional sensory neurons, most prominently ADL and AWB (Chao et al., 2004). However, exogenous TA or OA directly inhibit responses to $100 \%$ octanol in wild-type animals in the absence of both food and exogenous 5-HT (Fig. 9). More importantly, these responses are mediated by additional TA and OA receptors apparently not involved in responses to dilute octanol. For example, TA still inhibits responses to $100 \%$ octanol in tyra-3(ok325); tyra2(tm1846); ser-2(pk1357) triple null mutants, and OA still inhibits responses in ser-3(ad1774); f14d12.6(ok371) double null animals. Presumably, these unidentified TA and OA receptors (or ligand-gated ion channels?) or combinations of receptors are found in neuronal circuits leading from the additional sensory neurons involved in responses to $100 \%$ octanol. Indeed, the interaction of multiple DA receptors in the modulation of dopaminergic signaling has been observed previously (Chase et al., 2004).

\section{Preexposure to exogenous TA or OA abolishes TA/OA} inhibition of 5-HT-dependent increases in octanol sensitivity To test whether preexposure to either TA or OA could alter octanol sensitivity, animals were incubated in TA or OA for $6 \mathrm{~h}$ before assay in either 100 or $30 \%$ octanol (for assay protocol, see Fig. $10 \mathrm{~A}$ ). As predicted, responses to $100 \%$ octanol after preexposure to either TA or OA demonstrated that animals desensitized and that the desensitization was amine-specific; i.e., preincubation in TA abolished responses to TA, but not OA, and preincubation in OA abolished responses to OA, but not TA (Fig. $10 B)$.

In contrast, responses to $30 \%$ octanol after preexposure to TA or OA were more complex (Fig. 10C). For example, exposure to TA dramatically increased sensitivity to dilute octanol in animals off food to levels observed in animals in the presence of bacteria or 5 -HT (Fig. $10 B$ ). These results suggest that preincubation with TA downregulates tyraminergic signaling and that TA physiologically inhibits wild-type responses to dilute octanol, or alternatively, TA exposure could have more peripheral effects, such as the upregulation of serotonergic signaling. In addition, preincubation in TA abolished both the TA and OA inhibition of 5-HTdependent increases in sensitivity to dilute octanol, suggesting that preexposure to TA had downregulated both tyraminergic and octopaminergic signaling. In contrast, preexposure to OA had no effect on sensitivity to dilute octanol in wild-type animals off food or in the presence of 5-HT. However, preincubation in OA abolished the OA and, to a lesser extent, TA inhibition of 5-HT-dependent increases in sensitivity to dilute octanol (Fig. $10 C)$.

In contrast to the amine-specific desensitization responses to $100 \%$ octanol, the lack of amine specificity in responses to $30 \%$ octanol is unclear. Responses to dilute octanol are mediated by a single sensory neuron, the ASH, whereas responses to $100 \%$ octanol involve additional sensory neurons, including AWB and ADL and additional TA/OA receptors (Chao et al., 2004; present study). Interestingly, our pharmacological characterization of two C. elegans TA receptors (SER-2 and TYRA-2) suggests that these receptors can bind $\mathrm{OA}$, albeit at significantly lower concentrations than TA $(\sim 10$-fold $)$, at least after heterologous expres- 


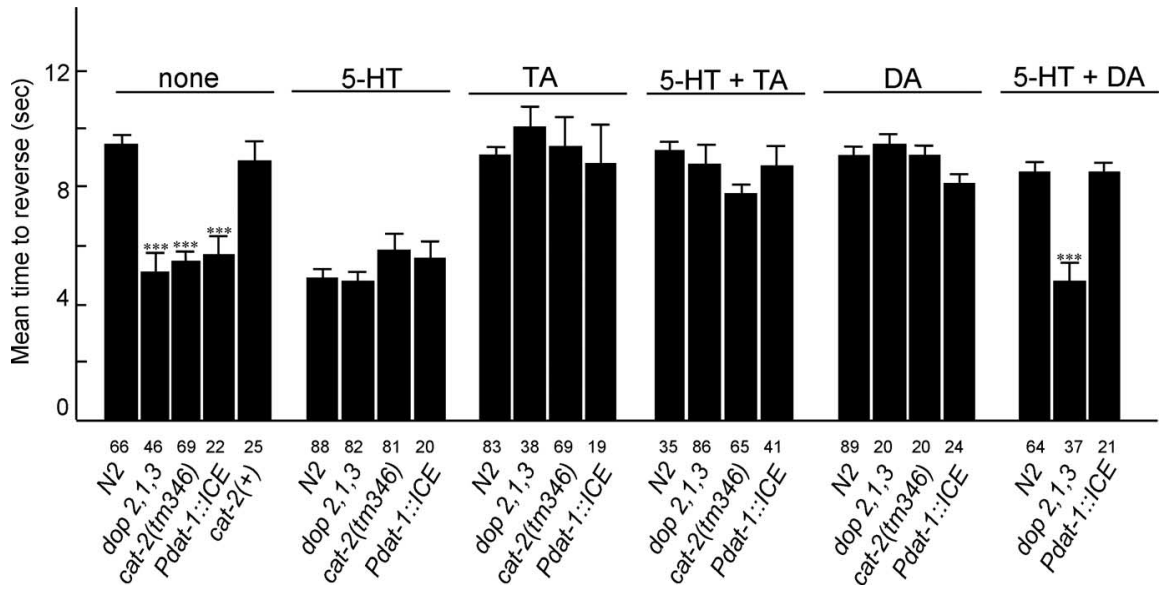

Figure 8. Dopamine does not appear to be involved in the tyramine inhibition of 5-HT-dependent responses to dilute octanol. Wild-type animals and mutant animals deficient in dopaminergic signaling were examined for their ability to respond to dilute octanol in the presence or absence of 5-HT, DA, and/or TA, as described in Materials and Methods. Data are presented as mean \pm $\mathrm{SE}(n)$ and analyzed by a two-tailed Student's $t$ test. ${ }^{* *} p<0.001$, significantly different from wild-type animals under identical incubation conditions. The number of trials is indicated under each bar.

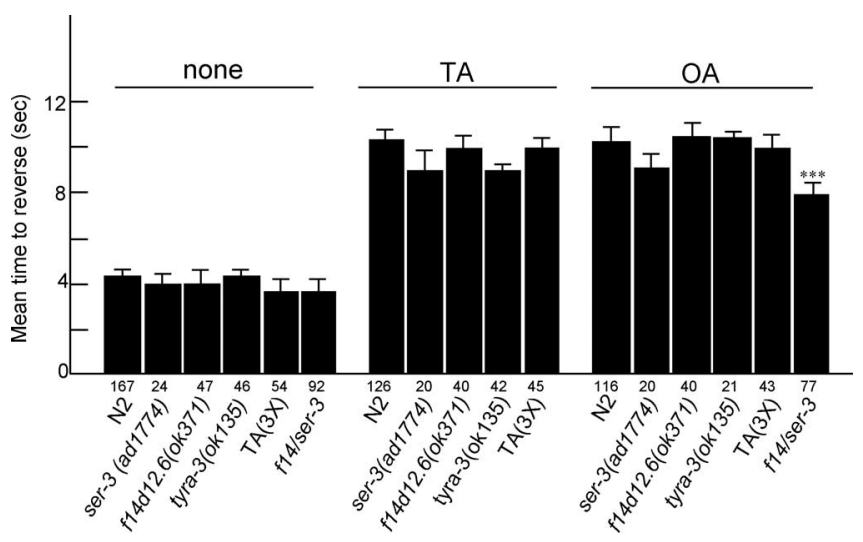

Figure 9. Tyramine and octopamine also inhibit responses to 100\% octanol. Wild-type and various TA/OA receptor null mutants were examined for responses to $100 \%$ octanol in the absence of food and in the presence of exogenous TA or $0 A$, as described in Materials and Methods. TA(3X), tyra-3(ok325); tyra-2(tm1846); ser-2(pk1357) triple null mutant; f14/ser-3, ser-3(ad1774); f14d12.6(ok371) double null mutant. Data are presented as mean \pm SE and analyzed by a two-tailed Student's $t$ test. ${ }^{* *} p<0.001$, significantly different from wild-type animals under identical incubation conditions. The number of trials is indicated under each bar.

sion. Similar observations have been reported for insect TA/OA receptors (Saudou et al., 1990; Vanden Broeck et al., 1995; Degen et al., 2000). Therefore, the preincubation conditions used in the present study may allow these ligands to accumulate to levels sufficient to activate the other receptors.

\section{Discussion}

In the present study, we have demonstrated that TA, OA, and DA all independently abolish 5-HT- and food-dependent increases in aversive responses to dilute octanol. However, although TA and OA both oppose the action of 5-HT, as has been observed for other 5-HT-dependent behaviors in C. elegans, their effects are mediated by distinct GPCRs; i.e., TYRA-3 mediates TA inhibition and F14D12.6 mediates OA inhibition of 5-HT-dependent responses to dilute octanol. The role of feeding status and 5-HT levels in the modulation of octanol avoidance has been demonstrated previously (Chao et al., 2004). When animals are on food, the ASHs are both necessary and sufficient for responses to dilute octanol. ASH-mediated aversive responses appear to be coupled to increases in intracellular $\mathrm{Ca}^{2+}$ through a pathway requiring the TRP channel subunit OSM-9 and the L-type calcium channel EGL-19 (Colbert et al., 1997; Hart et al., 1999; Hilliard et al., 2005). 5-HT increases responsiveness to dilute octanol through a pathway requiring GPA-11, a G-protein subunit expressed exclusively in the ASH and ADL sensory neurons, and also increases ASH-mediated $\mathrm{Ca}^{2+}$ transients, at least in response to nose touch (Chao et al., 2004; Hilliard et al., 2005). Together, these results suggest that a 5-HT receptor is expressed in the ASH that directly increases its sensitivity to dilute octanol. However, TA/OA/DA could function at multiple levels within the nervous system to inhibit the ASH-mediated pathways modulating locomotory behaviors. Indeed, the expression patterns of tyra-3::gfp and f14d12.6::gfp do appear to overlap significantly. F14D12.6 is expressed in the ASH, and the OA sensitivity of f14d12.6 null mutants can be rescued by the expression of F14D12.6 in the ASH under the control of the sra-6 promoter, suggesting that F14D12.6 directly alters ASH responsiveness. In contrast, the site of action of TYRA-3 is unclear, but it does not appear to involve the release of DA from dopaminergic neurons, where it is highly expressed. For example, TA still inhibits 5-HT-dependent increases in sensitivity to dilute octanol in cat-2(tm346) null animals that exhibit dramatically reduced DA levels, Pdat-1::ICE animals that lack dopaminergic neurons, and dop-2; dop-1; dop-3 mutants that lack three key DA receptors and fail to respond to DA in these assays (Chase et al., 2004; Sanyal et al., 2004; Nass et al., 2005).

In mammals, trace amines, such as TA/OA, have been implicated in a number of disorders, including depression, schizophrenia, and ADHD (Sandler et al., 1979; Davis and Boulton, 1994; Kusaga, 2002). Recently, a novel family of TAARs has been identified; however, in contrast to the classical BAs, much less is known about trace amine-mediated signaling. Both TAAR1 and TAAR2 bind TA and other ligands such as iodothyronamines, with high affinity and couple to $\mathrm{G} \alpha_{\mathrm{s}}$ after heterologous expression (Borowsky et al., 2001; Bunzow et al., 2001; Hart et al., 2006). Based on studies in TAAR1 knock-out mice, amphetamines also appear to be high-potency agonists at TAAR1 receptors, and TAAR1 appears to modulate catecholaminergic function, suggesting that signaling through TAARs might provide useful targets for the treatment of a variety of behavioral disorders (Wolinsky et al., 2006). In contrast, many of the TAARs (but not TAAR1) are selectively expressed in the olfactory epithelium and detect volatile amines found in the urine, suggesting a chemosensory function associated with the detection of social cues that is distinct from the much more abundant odorant receptors (Liberles and Buck, 2006).

All mammalian TAARs contain a consensus NSXXNPXX[Y$\mathrm{H}] \mathrm{XXX}[\mathrm{YF}] \mathrm{XWF}$ sequence in TM VII, and invertebrate genomes do not appear to contain sequences belonging to the TAAR family, despite the fact that TA/OA binding has been identified in several invertebrate species (Lindemann et al., 2005; Gloriam et al., 2005). In contrast to vertebrates, tyraminergic and octopaminergic signaling has been better characterized in invertebrates. Nematodes lack an autonomic nervous system and cannot syn- 
A Adaptation assay

Pre-incubation (+/- ligand)

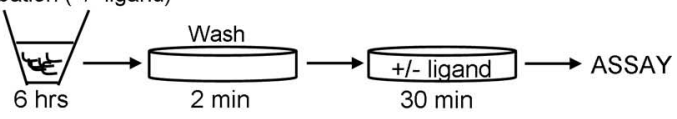

B Response to $100 \%$ octanol

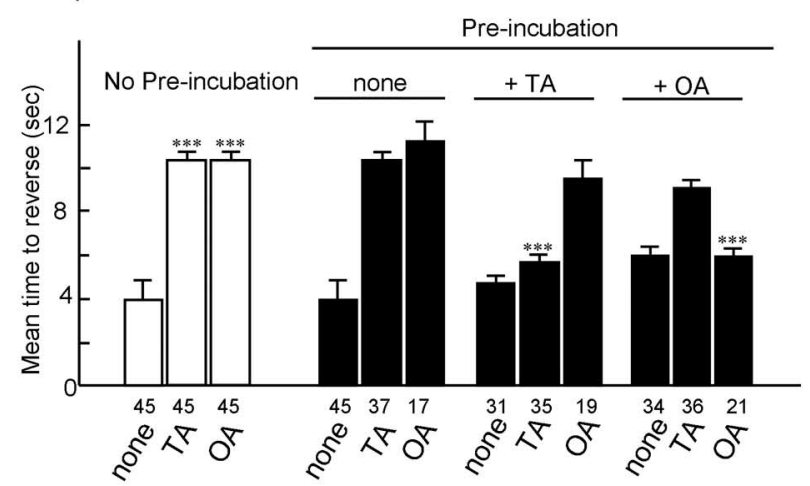

C Response to $30 \%$ octanol

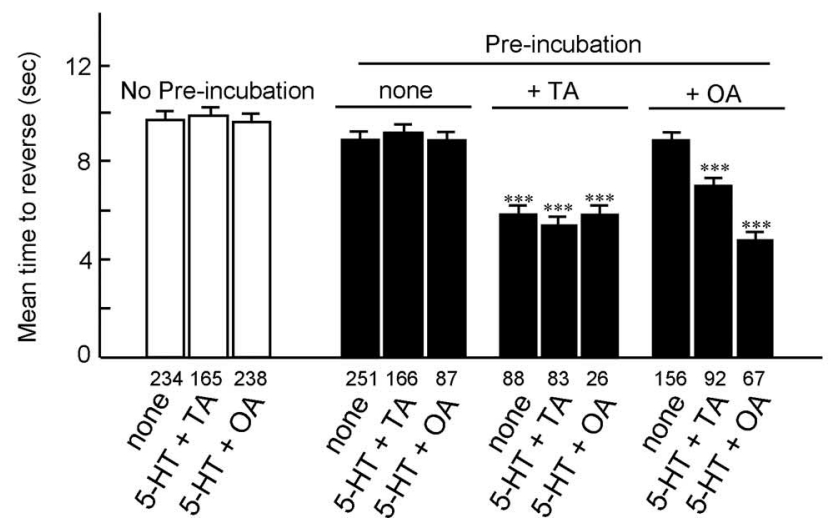

Figure 10. Effects of preincubation in either tyramine or octopamine on avoidance responses to 30 and $100 \%$ octanol. Wild-type animals were preincubated $(6 \mathrm{~h})$ in the presence or absence of TA or $0 \mathrm{~A}(4 \mathrm{~mm})$ and then assayed for responses to 30 and $100 \%$ octanol. $A$, Outline of "adaptation" protocol. B, Effects of TA or OA on sensitivity to $100 \%$ octanol after preincubation in either TA or OA. C, Effects of TA or OA on sensitivity to dilute (30\%) octanol after preincubation in either TA or $0 A$. Data are presented as mean $\pm S E$ and analyzed by a two-tailed Student's $t$ test. ${ }^{* * *} p<0.001$, significantly different from wild-type animals under identical incubation conditions. The number of trials is indicated under each bar.

thesize epinephrine/norepinephrine. Instead, nematodes use BAs, such as 5-HT, TA, and OA, to modulate most key processes, including feeding, locomotion, and egg laying. In general, TA and OA oppose the action of 5-HT. For example, in C. elegans, exogenous TA or OA cause hyperactivity and suppress 5-HTstimulated pharyngeal pumping or egg laying (Horvitz et al., 1982; Ségalat et al., 1995; Niacaris and Avery, 2003). This observation contrasts with recent reports from insects, in which tyraminergic and octopaminergic signaling often appear to function antagonistically. TA- and OA-specific neurons, receptors, signaling pathways, and phenotypes have been described in both insects (Saudou et al., 1990) and nematodes (Rex et al., 2002). In insects, TA and OA have opposite effects on a variety of behaviors, including locomotion, excitatory junction potentials in muscle strips, and chloride ion conductance across Malpighian tubules in Drosophila (Nagaya et al., 2002; Blumenthal, 2003; Saraswati et al., 2003). In general, insect GPCRs with a preference for TA couple to a decrease in cAMP levels, whereas GPCRs with a preference for OA couple to an elevation in cAMP levels (Roeder, 2005). However, an insect GPCR specific for TA that activates $\mathrm{Ca}^{2+}$-dependent chloride channels after expression in $\mathrm{Xe}$ nopus oocytes has recently been identified, making generalizations difficult (Cazzamali et al., 2005). Interestingly, all of the TA/OA receptors analyzed in the present study clustered based on documented G-protein coupling and not necessarily ligand specificity.

Similarly, a role for TA, independent of OA, has been uncovered in C. elegans by the identification of GPCRs with higher affinities for TA than OA and the examination of $t d c-1$ and $t b h-1$ mutants that are unable to synthesize OA/TA and OA, respectively. For example, $t d c-1$ is expressed in RIC and RIM neurons, and the UV1 and gonadal sheath cells, whereas $t b h-1$ is expressed only in the RICs and gonadal sheath cells, suggesting that the RICs release OA and the RIMs release TA and perhaps OA (Alkema et al., 2005). Most importantly, $t d c-1$, but not $t b h-1$, null mutants exhibit hyperactive egg laying and do not suppress head oscillations in response to anterior touch, suggesting a specific role for TA in these behaviors (Alkema et al., 2005). However, TA- and OA-dependent phenotypes have been examined only cursorily, and virtually nothing is known about the individual TA and OA receptors or neuronal circuits involved in the modulation of individual behaviors. Of the downstream interneurons innervated by the ASH, including the AIAs, AIBs, and RIAs, only the command interneurons that control the choice between forward and backward locomotion appear to be directly innervated by the RIMs or RICs. However, the TA/OA receptors expressed directly in the command interneurons remain to be identified. The identification of at least five distinct C. elegans GPCRs (SER-2, TYRA-2, TYRA-3, SER-3, and F14D12.6) capable of binding TA or OA with high affinity and the availability of null mutants for each of these receptors highlight the utility of $C$. elegans as a model to study the GPCR-mediated aminergic modulation of aversive behaviors. Indeed, the modulatory effects of TA and OA may be largely extrasynaptic, as has been suggested for dopaminergic signaling (Chase et al., 2004; Sanyal et al., 2004). However, it is unclear how TA/OA enters the pseudocoelomic fluid of the animals from either neuronal or other nonneuronal tissues, such as the gonadal sheath and UV1 cells, which also appear to be capable of TA and/or OA synthesis.

Responses to both TA and OA adapted to preexposure to the amines and, at least in the case of $100 \%$ octanol, were amine specific; i.e., preincubation in TA abolished responses to TA, but not $\mathrm{OA}$, and preincubation in $\mathrm{OA}$ abolished responses to OA, but not TA. Adaptation to the effects of BAs has been observed previously in $C$. elegans. For example, wild-type animals treated overnight with 5-HT fail to respond to 5-HT in egg-laying assays (Schafer and Kenyon, 1995; Carnell et al., 2005). Adaptation to chronic elevation in neurotransmitter levels often occurs by downregulation of either/both receptors and downstream signaling pathways. Whether the potential downregulation of aminergic modulation plays an adaptive role in C. elegans remains to be determined, but it may modulate olfactory responses according to long-term feeding status.

Together, the results of the present study suggest that 5-HT, TA, OA, and DA may all play a role in the modulation of aversive olfactory responses and highlight the complexity of the aminergic pathways linking sensory input with locomotory behaviors. For example, the levels of these amines change with nutritional state and have the capacity to differentially modulate the distinct sensory-mediated, food-dependent foraging strategies that have been identified previously (Sawin et al., 2000; Tsalik et al., 2003; 
Gray et al., 2004). These observations highlight the utility of the C. elegans model system for studying the aminergic modulation of sensory output and could have broad application to higher organisms. These studies are continuing with the goal of identifying the roles of the individual neurons involved in the aminedependent, locomotory circuit modulating aversive olfactory responses. For example, with the availability of ASH-specific promoters and the rapidly growing number of C. elegans signaling mutants, we can directly examine the signaling pathways potentially coupled to F14D12.6 stimulation in the ASHs, their interaction with other ASH inputs, and their ability to modulate ASH downstream signaling.

\section{References}

Alkema M, Hunter-Ensor M, Ringstad N, Horvitz R (2005) Tyramine functions independently of octopamine in the Caenorhabditis elegans nervous system. Neuron 46:247-260.

Almaula N, Ebersole BJ, Zhang D, Weinstein H, Sealfon S (1996) Mapping the binding site pocket of the 5-hydroxytryptamine2A receptor. J Biol Chem 271:14672-14675.

Bargmann CI, Hartwieg E, Horvitz HR (1993) Odorant-selective genes and neurons mediate olfaction in C. elegans. Cell 74:515-527.

Berke JD, Hyman SE (2000) Addiction, dopamine, and the molecular mechanisms of memory. Neuron 25:515-532.

Blumenthal EM (2003) Regulation of chloride permeability by endogenously produced tyramine in the Drosophila Malpighian tubule. Am J Physiol Cell Physiol 284:718-728.

Borowsky B, Adham N, Jones KA, Raddatz R, Artymyshyn R, Ogozalek KL, Durkin MM, Lakhlani PP, Bonini JA, Pathirana S, Boyle N, Pu X, Kouranova E, Lichtblau H, Ochoa FY, Branchek TA, Gerald C (2001) Trace amines: identification of a family of mammalian $\mathrm{G}$ protein-coupled receptors. Proc Natl Acad Sci USA 98:8966-8971.

Brenner S (1974) The genetics of Caenorhabditis elegans. Genetics 77:71-94.

Bunzow JR, Sonders MS, Arttamangkul S, Harrison LM, Zhang GE, Quigley DL, Darland T, Suchland KL, Pasumamula S, Kennedy JL, Olson SB, Magenis RE, Amara SG, Grandy DK (2001) Amphetamine, 3,4methylenedioxymethamphetamine, lysergic acid diethylamide, and metabolites of the catecholamine neurotransmitters are agonists of a rat trace amine receptor. Mol Pharmacol 60:1181-1188.

Carnell L, Illi J, Hong W, McIntire SL (2005) The G-protein-coupled serotonin receptor SER-1 regulates egg laying and male mating behaviors in Caenorhabditis elegans. J Neurosci 25:10671-10681.

Carre-Pierrat M, Maillie D, Johnsen R, Hyde R, Hart A, Granger L, Ségalat L (2006) Characterization of the Caenorhabditis elegans $\mathrm{G}$ protein-coupled serotonin receptors. Invert Neurosci 4:189-205.

Cazzamali G, Klaerke D, Grimmelikhuijzen C (2005) A new family of insect tyramine receptors. Biochem Biophys Res Commun 338:1189-1196.

Chao MY, Komatsu H, Fukuto HS, Dionne HM, Hart AC (2004) Feeding status and serotonin rapidly and reversibly modulate a Caenorhabditis elegans chemosensory circuit. Proc Natl Acad Sci USA 101:15512-15517.

Chao MY, Larkins-Ford J, Tucey TM, Hart A (2005) lin-12 Notch functions in the adult nervous system of C. elegans. BMC Neurosci 6:45.

Chase DL, Pepper JS, Koelle MR (2004) Mechanism of extrasynaptic dopamine signaling in Caenorhabditis elegans. Nat Neurosci 7:1096-1103.

Choudhary MS, Craig S, Roth BL (1993) A single point mutation of a conserved phenylalanine abolishes $4[125 \mathrm{I}]$ iodo-(2,5 dimethoxy) phenylisopropylamine and $[3 \mathrm{H}]$-mesulergine but not $[3 \mathrm{H}]$-ketanserin binding to 5HT 2 receptors. Mol Pharmacol 43:755-761.

Colbert H, Smith T, Bargmann C (1997) OSM-9, a novel protein with structural similarity to channels, is required for olfaction, mechanosensation, and olfactory adaptation in Caenorhabditis elegans. J Neurosci $17: 8259-8269$

Davis BA, Boulton AA (1994) The trace amines and their acidic metabolites in depression-an overview. Prog Neuropsychopharmacol Biol Psychiatry 18:17-45.

de Bono M, Maricq AV (2005) Neuronal substrates of complex behaviors in C. elegans. Annu Rev Neurosci 28:451-501.

Degen J, Gewecke M, Roeder T (2000) Octopamine receptors in the honey bee and locust nervous system: pharmacological similarities between homologous receptors of distantly related species. Br J Pharmacol 130:587-594.
Duvernay MT, Zhou F, Wu G (2004) A conserved motif for the transport of $G$ protein-coupled receptors from the endoplasmic reticulum to the cell surface. J Biol Chem 279:30741-30750.

Gloriam DEI, Bjarnadottir TK, Schiöth HB, Fredriksson R (2005) High species variation within the repertoire of trace amine receptors. Ann NY Acad Sci 1040:323-327.

Gray JM, Karow DS, Lu H, Chang AJ, Chang JS, Ellis RE, Marletta MA, Bargmann CI (2004) Oxygen sensation and social feeding mediated by a C. elegans guanylate cyclase homologue. Nature 430:317-322.

Hamdan FF, Ungrin MD, Abramovitz M, Ribeiro P (1999) Characterization of a novel serotonin receptor from Caenorhabditis elegans: cloning and expression of two splice variants. J Neurochem 72:1372-1383.

Hart AC, Kass J, Shapiro JE, Kaplan JM (1999) Distinct signaling pathways mediate touch and osmosensory responses in a polymodal sensory neuron. J Neurosci 19:1952-1958.

Hart ME, Suchland KL, Miyakawa M, Bunzow JR, Grandy DK, Scalan TS (2006) Trace amine-associated receptor agonists: synthesis and evaluation of thyronamines and related analogues. J Med Chem 49:1101-1112.

Hilliard M, Apicella A, Kerr R, Suzuki H, Bazzicalupo, Schafer W (2005) In vivo imaging of C. elegans ASH neurons: cellular response and adaptation to chemical repellents. EMBO J 24:63-73.

Hobert O (2002) PCR fusion-based approach to create reporter gene constructs for expression analysis in transgenic C. elegans. Biotechniques 32:728-730.

Hobson RJ, Geng J, Gray AD, Komuniecki RW (2003) SER-7b, a constitutively active $\mathrm{G} \alpha_{\mathrm{s}}$ coupled 5- $\mathrm{HT}_{7}$-like receptor expressed in the Caenorhabditis elegans M4 pharyngeal motorneuron. J Neurochem 87:22-29.

Horvitz HR, Chalfie M, Trent C, Sulston JE, Evans PD (1982) Serotonin and octopamine in the nematode Caenorhabditis elegans. Science 216:1012-1014.

Huang X, Xiao H, Rex EB, Hobson RJ, Messer WS, Komuniecki PR, Komuniecki RW (2002) Functional characterization of alternatively spliced $5-\mathrm{HT}_{2}$ receptor isoforms from the pharynx and muscle of the parasitic nematode, Ascaris suum. J Neurochem 83:249-258.

Kramer JM, French RP, Park E, Johnson JJ (1990) The Caenorhabditis elegans rol-6 gene, which interacts with the sqt- 1 collagen gene to determine organismal morphology, encodes a collagen. Mol Cell Biol 10:2081-2089.

Kubiak TM, Larsen MJ, Nulf SC, Zantello MR, Burton KJ, Bowman JW, Modric T, Lowery DE (2003) Differential activation of "social" and "solitary" variants of the Caenorhabditis elegans $\mathrm{G}$ protein-coupled receptor NPR-1 by its cognate ligand AF9*. J Biol Chem 278:33724-33729.

Kusaga A (2002) Decreased beta-phenylethylamine in urine of children with attention deficit hyperactivity disorder and autistic disorder (in Japanese). No To Hattatsu 34:243-248.

Lackner MR, Nurrish SJ, Kaplan JM (1999) Facilitation of synaptic transmission by EGL-30 Gqalpha and EGL-8 PLCbeta: DAG binding to UNC-13 is required to stimulate acetylcholine release. Neuron 24:335-346.

Liberles SD, Buck LB (2006) A second class of chemosensory receptors in the olfactory epithelium. Nature 442:645-650.

Lindemann L, Ebeling M, Kratochwil NA, Bunzow JR, Grandy DK, Hoener MC (2005) Trace amine associated receptors form structurally and functionally distinct subfamilies of novel G protein-coupled receptors. Genomics 85:372-385.

Lucki I (1998) The spectrum of behaviours influenced by serotonin. Biol Psychiatry 44:51-162.

Mello C, Fire A (1995) DNA transformation. In: Methods in cell biology, Vol 48, Caenorhabditis elegans. Modern biological analysis of an organism. (Epstein HF, Shaker DC, eds), pp. 452-482. San Diego: Academic.

Mendel JE, Korswagen HC, Liu KS, Hajdu-Cronin YM, Simon MI, Plasterk RH, Sternberg PW (1995) Participation of the protein Go in multiple aspects of behavior in C. elegans. Science 267:1652-1655.

Miller KG, Emerson MD, Rand JB (1999) Goalpha and diacylglycerol kinase negatively regulate the Gqalpha pathway in C. elegans. Neuron 24:323-333.

Nagaya Y, Kutsukake M, Chigusa SI, Komatsu A (2002) A trace amine, tyramine, functions as a neuromodulator in Drosophila melanogaster. Neurosci Lett 329:324-328.

Näsman J, Kukkonen JP, Åkerman KEO (2002) Dual signalling by different octopamine receptors converges on adenylate cyclase in Sf 9 cells. Insect Biochem Mol Biol 32:285-293.

Nass R, Hahn MK, Jessen T, McDonald PW, Carvelli L, Blakel Y (2005) A 
genetic screen in Caenorhabditis elegans for dopamine neuron insensitivity to 6-hydroxydopamine identifies dopamine transporter mutants impacting transporter biosynthesis and trafficking. J Neurochem 94:774-785.

Niacaris T, Avery L (2003) Serotonin regulates repolarization of the C. elegans pharyngeal muscle. J Exp Biol 206:223-231.

Nurrish S, Ségalat L, Kaplan JM (1999) Serotonin inhibition of synaptic transmission: Galpha(0) decreases the abundance of UNC-13 at release sites. Neuron 24:231-242.

Olde B, McCombie WR (1997) Molecular cloning and functional expression of a serotonin receptor from Caenorhabditis elegans. J Mol Neurosci 8:53-62.

Rex E, Komuniecki R (2002) Characterization of a tyramine receptor from Caenorhabditis elegans. J Neurochem 82:1352-1359.

Rex E, Molitor SC, Hapiak V, Xiao H, Henderson M, Komuniecki R (2004) Tyramine receptor (SER-2) isoforms are involved in the regulation of pharyngeal pumping and foraging behavior in Caenorhabditis elegans. J Neurochem 91:1104-1115.

Rex E, Hapiak V, Hobson R, Smith K, Xiao H, Komuniecki R (2005) tyra-2 (F01E11.5): a Caenorhabditis elegans tyramine receptor expressed in the MC and NSM pharyngeal neurons. J Neurochem 94:181-191.

Roeder T (2005) Tyramine and octopamine: ruling behavior and metabolism. Anu Rev Entomol 50:447-477.

Roth BL, Shoham MS, Choudhary MS, Khan N (1997) Identification of conserved aromatic residues essential for agonist binding and second messenger production at 5-hydroxytryptamine $2 \mathrm{~A}$ receptors. Mol Pharmacol 52:259-266.

Sandler M, Ruthven CR, Goodwin BL, Reynolds GP, Rao VA, Coppen A (1979) Deficient production of tyramine and octopamine in cases of depression. Nature (Lond) 278:357-358.

Sanyal S, Wintle R, Kindt KS, Nuttley WM, Arvan R, Fitzmaurice P, Bigras E, Merz DC, Hébert E, van der Kooy D, Schafer WR, Culotti JG, Van Tol HHM (2004) Dopamine modulates the plasticity of mechanosensory responses in Caenorhabditis elegans. EMBO J 23:473-482.

Saraswati S, Fox LE, Soll DR, Wu C (2003) Tyramine and octopamine have opposite effects on the locomotion of Drosophila larvae. J Neurobiol $58: 425-441$.
Saudou F, Amlaiky N, Plassat J, Borelli E, Hen R (1990) Cloning and characterization of a Drosophila tyramine receptor. EMBO J 9:3611-3617.

Sawin ER, Ranganathan R, Horvitz HR (2000) C. elegans locomotory rate is modulated by the environment through a dopaminergic pathway and by experience through a serotonergic pathway. Neuron 26:619-631.

Schafer WR, Kenyon CJ (1995) A calcium-channel homologue required for adaptation to dopamine and serotonin in Caenorhabditis elegans. Nature 375:73-78.

Ségalat L, Elkes DA, Kaplan M (1995) Modulation of serotonin-controlled behaviors by $\mathrm{G}_{\mathrm{O}}$ in Caenorhabditis elegans. Science 267:1648-1651.

Strader CD, Candelore MR, Hill WS, Sigal IS, Dixon AF (1989) Identification of two serine residues involved in agonist activation of the b-adrenergic receptor. J Biol Chem 264:13572-13578.

Suo S, Sasagawa N, Ishiura S (2002) Identification of a dopamine receptor from Caenorhabditis elegans. Neurosci Lett 319:13-16.

Suo S, Sasagawa N, Ishiura S (2003) Cloning and characterization of a Caenorhabditis elegans D2-like dopamine receptor. J Neurochem 86:869-878.

Suo S, Kimura Y, Vab Tol HM (2006) Starvation induces cAMP response elements-binding protein-dependent gene expression through octopamine-Gq signaling in Caenorhabditis elegans. J Neurosci 26:10082-10090.

Troemel ER, Chou JH, Dwyer ND, Colbert HA, Bargmann CI (1995) Divergent seven transmembrane receptors are candidate chemosensory receptors in C. elegans. Cell 83:207-218.

Tsalik EL, Niacaris T, Wenick AS, Pau K, Avery L, Hobert O (2003) LIM homeobox gene-dependent expression of biogenic amine receptors in restricted regions of the C. elegans nervous system. Devel Biol 263:81-102.

Vanden Broeck J, Vulsteke V, Huybrechts R, De Loof A (1995) Characterization of a cloned locust tyramine receptor cDNA by functional expression in permanently transformed Drosophila S2 cells. J Neurochem 64:2387-2395.

Wolinsky TD, Swanson CJ, Smith KE, Zhong H, Borowsky B, Seeman P, Branchek T, Gerald CP (2006) The trace amine 1 receptor knockout mouse: an animal model with relevance to schizophrenia. Genes Brain Behav 6:628-639. 